\title{
MEMS Hydrogen Gas Sensor for the Entire Concentration Range of Hydrogen Gas
}

\author{
Mitsuteru Kimura* and Noriaki Takashima \\ Graduate School of Engineering, Tohoku Gakuin University, \\ 1-13-1 Chuo, Tagajo, Miyagi 985-8537, Japan
}

(Received January 6, 2011; accepted April 12, 2011)

Key words: hydrogen sensor, MEMS, exothermic reaction, thermal conductivity, cantilever, SOI

We have proposed a new type of hydrogen gas sensor that is a combination of two types of hydrogen sensor, in which one is for a lower hydrogen gas concentration of less than $4 \%$ and the other is for a higher hydrogen gas concentration of more than $4 \%$. The hydrogen gas sensor for low concentration works basically at room temperature based on the exothermic reaction of a Pd film formed on the cantilever and that for high concentration works during heating to about $150^{\circ} \mathrm{C}$ as a thermal conductance-type sensor by using the high thermal conductivity of hydrogen gas. This hydrogen gas sensor with a cantilever as a sensing area is fabricated using micro-electromechanical systems (MEMS) technology and silicon-on-insulator (SOI) substrate. A nichrome (NiCr) microheater and three thin-film thermocouples composed of an SOI layer of heavily doped n-type impurity and a Ni film layer are formed on the cantilever, and two areas, namely, a sensing area (region $\mathrm{B}_{\mathrm{S}}$ ) with the $\mathrm{Pd}$ film and a reference area (region $\mathrm{B}_{\mathrm{R}}$ ) without the $\mathrm{Pd}$ film, are formed with the same size in the vicinity of the tip of the cantilever. We have demonstrated that the proposed hydrogen gas sensor can measure hydrogen concentration at a very wide range from 0.1 to $100 \%$ within about $10 \mathrm{~s}$.

\section{Introduction}

Hydrogen gas is focused on as a new energy source and as a carrier gas in metal organic chemical vapor deposition (MOCVD) for semiconductor devices. Since hydrogen has an explosion risk at a volume ratio of about $4 \%$ (vol. \%) in air, a hydrogen gas sensor for low-concentration hydrogen gas detection of less than about $4 \%$ is necessary to ensure safety. On the other hand, a high-concentration hydrogen gas sensor is also necessary to control its concentration for carrier gas and fuel.

"Corresponding author: e-mail: kimura@tjcc.tohoku-gakuin.ac.jp 
Various types of hydrogen gas sensor based on different principles have been studied. Traditionally, the contact combustion-type hydrogen gas sensor has been well known..$^{(1,2)}$ In these hydrogen gas sensors, hydrogen is burned by a heater with a catalyst, such as Pt heated to about $100^{\circ} \mathrm{C}$ or more. The temperature difference between the sensing area and the substrate is measured using a thermopile. However, a relatively high temperature is needed to achieve the contact combustion in the atmosphere containing oxygen. Since the sensitivity to hydrogen gas depends on the state of the catalyst surface, we have faced problems such as a change in the catalyst surface over time, selectivity to flammable gas such as methane, and the formation reproducibility of the catalyst surface.

A hydrogen gas sensor using the metal-oxide-semiconductor field-effect transistor (MOSFET), which has the catalyst on the gate oxide and is based on the mechanism of the change in the work function including the Nernstian behavior, was reported. ${ }^{(3-6)}$ This sensor must be heated, such as to about $50^{\circ} \mathrm{C}$, by a heater owing to a decrease in the effects of ambient temperature and humidity, and to expedite the response. However, it had a problem in terms of the relatively large power consumption required to heat it up because of the large heat capacity of the sensor chip.

We have proposed a cantilever-type hydrogen gas sensor formed by MEMS technology, which is based on the exothermic reaction at the occlusion of hydrogen and the selectivity of the Pd film occluding only hydrogen. ${ }^{(7)}$

In this study, the hydrogen gas sensor composed of a cantilever having a microheater and three thermocouples formed by MEMS technology, which can cover the measurement of the entire hydrogen gas concentration range $(0.1-100 \%)$ with only one sensor, is proposed and demonstrated.

For a low hydrogen concentration of $4 \%$ or less (the low-hydrogen-concentration mode), we use the exothermic reaction at the occlusion of hydrogen and hydrogen selectivity of the Pd film to the other gases operating at near room temperature. On the other hand, for a high hydrogen concentration of $4 \%$ or more (the high-hydrogenconcentration mode), we use the sensor as a thermal conductance-type sensor since hydrogen gas has the highest thermal conductivity of all gases.

\section{Materials and Methods}

\subsection{Structure and mechanism of hydrogen gas sensor}

In Fig. 1, a photograph of the fabricated hydrogen gas sensor is shown. This hydrogen gas sensor is composed of an SOI cantilever having regions $\mathrm{A}$ and $\mathrm{B}$, and region $B$ is divided into two areas: region $B_{S}$ (sensing area) and region $B_{R}$ (reference area) with the same size. In this prototype hydrogen sensor, the SOI cantilever is about $5 \mathrm{~mm}$ long. In region $\mathrm{B}_{\mathrm{S}}$, a Pd film of about $1 \mu \mathrm{m}$ thickness as a hydrogen occlusion material is deposited by RF sputtering to occlude hydrogen, while no Pd film is present in region $\mathrm{B}_{\mathrm{R}}$. A film composed of Ti/Ni/Ti (Ti: $\sim 0.05 \mu \mathrm{m}$ thick; $\mathrm{Ni}: \sim 0.5 \mu \mathrm{m}$ thick), which is also used as one of the thermoelectric materials for a thin-film thermocouple, underneath the Pd film is deposited to increase adhesion with the $\mathrm{SiO}_{2}$ film and to relieve the tension of the Pd film occluded by hydrogen, because the Pd film has a tendency to peel when exposed to hydrogen gas. In region A, which is located in the vicinity of the 


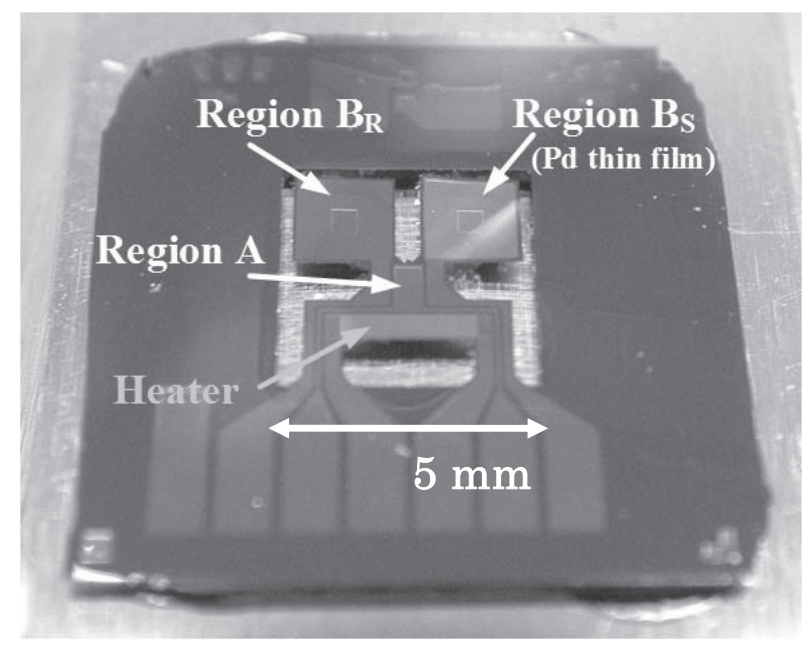

Fig. 1. Photograph of the fabricated hydrogen gas sensor.

center of the cantilever, a $\mathrm{NiCr}$ film as a microheater is formed and region $\mathrm{B}$, including regions $B_{S}$ and $B_{R}$, is located near the tip of the cantilever.

In regions $\mathrm{A}, \mathrm{B}_{\mathrm{S}}$, and $\mathrm{B}_{\mathrm{R}}$, hot junctions of the thermocouple are formed, and their cold junctions are formed in the $\mathrm{Si}$ substrate (Si chip). These thermocouples are composed of thermoelectric materials of a heavily n-type impurity-doped SOI (phosphorous $\mathrm{P}$, $1,100^{\circ} \mathrm{C}, 1 \mathrm{~h}$, thermal diffusion) and a Ni film (about $0.5 \mu \mathrm{m}$ thick) formed on it via a thermally grown $\mathrm{SiO}_{2}$ film (about $1 \mu \mathrm{m}$ thick). These thermocouples have a Seebeck constant of about $-160 \mu \mathrm{V} / \mathrm{K}$. The Ni film is sandwiched by very thin Ti films, as Ti/Ni/ $\mathrm{Ti}$, deposited by RF sputtering to increase adhesion with the $\mathrm{SiO}_{2}$ film and to prevent the overetching of the Ni film during its patterning.

Figure 2 shows a diagrammatic illustration of the measurement circuit of the hydrogen gas sensor. The total resistance of series-connected thermocouples to measure the temperature difference $\Delta T b$ between regions $B_{S}$ and $B_{R}$ was about $33 \Omega$. In this measurement circuit, the temperature of region $A$ can also be measured by using thermocouple A by turning on the switch for thermocouples. The feedback resistance $R_{\mathrm{f}}$ is $100 \mathrm{k} \Omega$ in the OP amplifier circuit shown in Fig. 2. We can obtain the amplified output voltage $V_{0}$ corresponding to the temperature difference $\Delta T b$ between region $\mathrm{B}_{\mathrm{S}}$, where the temperature rises by exothermic reaction when occluding hydrogen in the $\mathrm{Pd}$ film, and region $B_{R}$, where no thermal reaction occurs, by using thermocouples formed in these regions.

In our proposed hydrogen sensor working at room temperature, the exothermic reaction will stop when the $\mathrm{Pd}$ film is saturated by occluded hydrogen, and then the temperature will decrease. This will lead to a peak in the output characteristics. It is necessary to discharge hydrogen from the Pd film periodically, after hydrogen is 


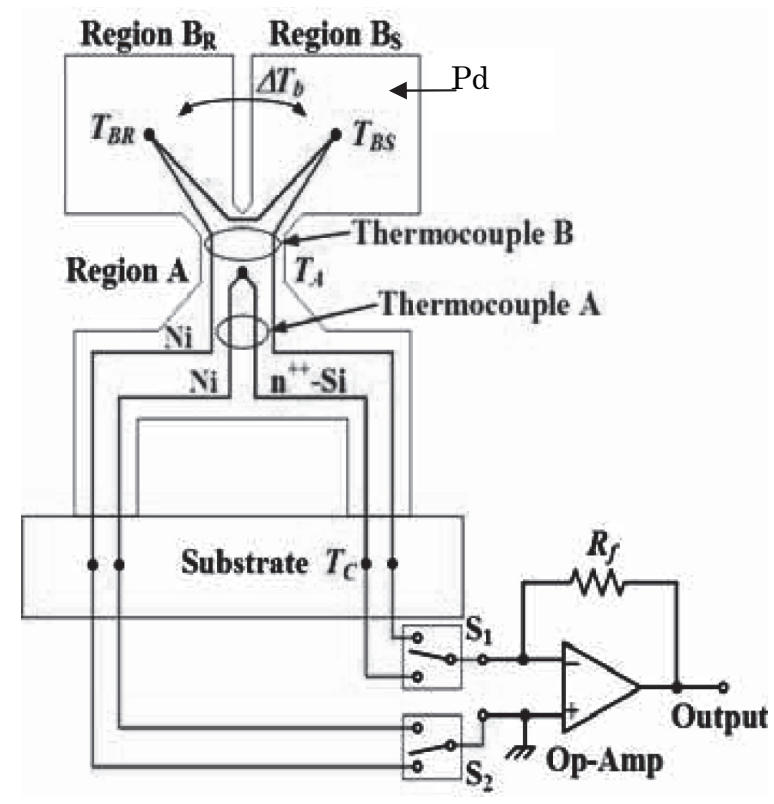

Fig. 2. Diagrammatic illustration of the measurement circuit of the hydrogen gas sensor.

occluded. In these sensor measurements, the hydrogen gas sensor is initialized to discharge the occluded hydrogen from the Pd film by heating the microheater to about $150^{\circ} \mathrm{C}$ by applying a rectangular waveform voltage for $5 \mathrm{~s}$ with an electric power of about $255 \mathrm{~mW}$.

First, we had planned to measure the hydrogen gas concentration only during the heating cycle by measuring the temperature difference $\Delta T b$ between region $B_{S}$ with a Pd film and region $B_{R}$ without a Pd film, because the Pd film will increase its temperature exothermically when hydrogen is occluded. However, we have found that the output voltage $V_{0}$ corresponding to the temperature difference $\Delta T b$ is decreased when the hydrogen concentration exceeds 4\%, as shown in Fig. 3. The reason is that the temperature of the cantilever will decrease under the same power consumption of the microheater because of the thermal conduction to a surrounding gas as the concentration of hydrogen gas, which has the highest thermal conductivity of all gases, increases.

In the low hydrogen concentration of less than $4 \%$, we have found that it is better to measure the hydrogen concentration during the cooling cycle, after the heating to discharge hydrogen from the Pd film is stopped. In this cooling cycle, we can apply the null detection method, which is well known as a highly precise measurement.

Thus, we have decided to adopt two measurements methods for lower and higher hydrogen concentrations with different principles: one is in a hydrogen concentration lower than about 4\% (the low-hydrogen-concentration mode), and the other is in a hydrogen concentration higher than about $4 \%$ (the high-hydrogen-concentration mode). 


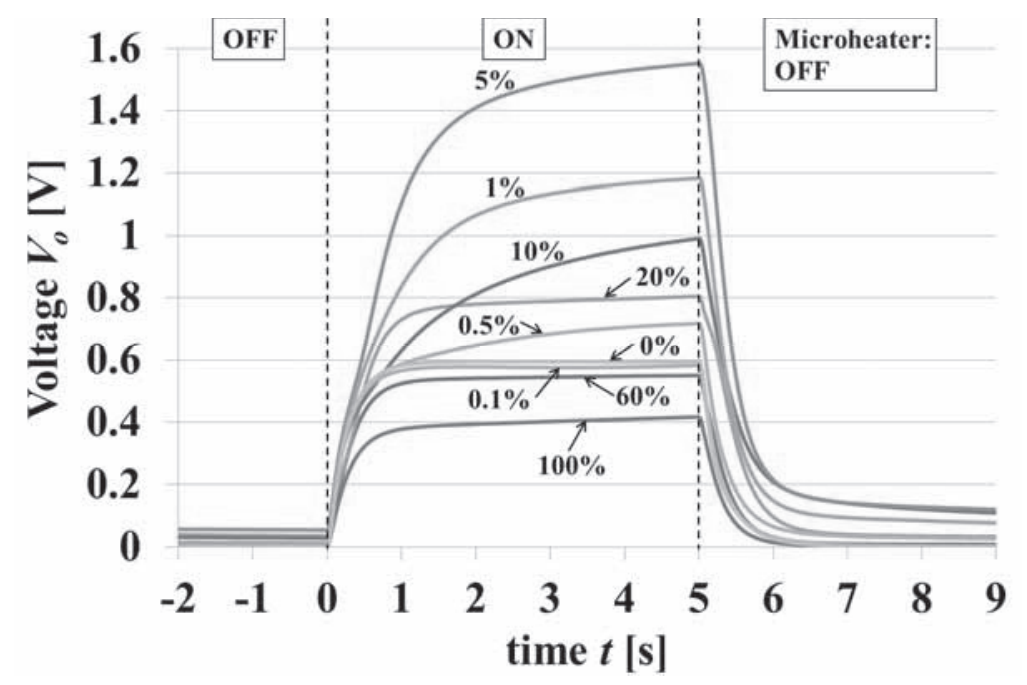

Fig. 3. Waveforms of output voltage $V_{0}$ corresponding to the temperature difference $\Delta T b$ of region $B_{S}$ (sensing area) and region $B_{R}$ (reference area) for various hydrogen concentrations in air when the microheater is heated by a constant rectangular waveform voltage.

For the low hydrogen concentration, we will measure the temperature difference $\Delta T b$ between $\mathrm{B}_{\mathrm{S}}$ and $\mathrm{B}_{\mathrm{R}}$ during the cooling cycle after stopping the heating. On the other hand, for the high hydrogen concentration, we will measure the temperature of region $\mathrm{A}$ based on that at zero hydrogen concentration during heating as a thermal conductancetype sensor. Since we have always carried out both measurement methods during heating and cooling cycles, we can compare these two output voltages and we can judge the real hydrogen concentration.

\subsection{Measurement in low-hydrogen-concentration mode}

A hydrogen storage metal such as palladium (Pd) will generate a metal hydride when reacting with hydrogen. In the thermal equilibrium state of the hydrogen storage metal, the reaction of hydrogen occlusion will be given by ${ }^{(8)}$

$$
\mathrm{Pd}+\mathrm{H}_{2} \rightleftharpoons \mathrm{PdH}_{x} \pm \Delta H,
$$

where $\Delta H$ is the heat of the reaction, and $x$ is the amount of occluded hydrogen.

The principle of hydrogen gas $\left(\mathrm{H}_{2}\right)$ sensing in this low-concentration region is that we measure a small temperature change based on the exothermic reaction when a material such as Pd occludes hydrogen, and let the temperature change correspond to the hydrogen concentration in air by calibration.

To detect the minute temperature change, we have fabricated a free-standing cantilever structure with a small thermal capacity using Si-MEMS technology, as shown 
in Fig. 1, so as to achieve very high temperature sensitivity.

We measure the hydrogen concentration during the cooling cycle after stopping the heating of a microheater formed on the cantilever in air including hydrogen. In this process, region $B_{R}$ will immediately return to room temperature as an ambient temperature because it has no $\mathrm{Pd}$ film on it, while the temperature in region $\mathrm{B}_{\mathrm{S}}$ will remain higher than that of region $B_{R}$ because of the exothermic reaction based on the occlusion of hydrogen in the Pd film, which will last until occlusion in the Pd film is saturated. Therefore, the measurement is executed at the time when it seems that the output voltage, corresponding to the temperature difference between region $\mathrm{B}_{\mathrm{R}}$ and the $\mathrm{Si}$ substrate (chip) being at room temperature, becomes almost zero.

General metallic alloys for hydrogen storage are easily oxidized and so cannot be used in air. However, a Pd film of a noble metal as a hydrogen occlusion material works with stability even at a high temperature in air. Thus, the Pd film will be the best material for the occlusion and discharge cycles of hydrogen.

\subsection{Measurement in high-hydrogen-concentration mode}

The thermal conductivity of hydrogen gas is the largest among gases. Therefore, as shown in Fig. 3, the heat radiation from the microheater increases as the hydrogen concentration in air is increased above $4 \%$ because of the effect of the thermal conductivity even if the microheater is heated by the same electric power, and the temperature decreases more than the temperature of the heater when heating it in air.

In Fig. 3, waveforms of output voltage $V_{0}$ corresponding to the temperature difference $\Delta T b$ for various hydrogen concentrations in air are shown, when the microheater is heated by a constant rectangular waveform voltage. In the low hydrogen concentration of $4 \%$ or less, we have adopted the measurement of the temperature difference $\Delta T b$ between $\mathrm{B}_{\mathrm{S}}$ and $\mathrm{B}_{\mathrm{R}}$; however, we cannot use this experimental method for the high hydrogen concentration, as shown in Fig. 3. Therefore, for the high hydrogen concentration above $4 \%$, we have adopted the other experimental method to measure the temperature difference using thermocouple A between region A, where the microheater is formed, and the Si substrate working as a thermal conductance-type sensor. We let the output voltage of thermocouple A correspond to each hydrogen concentration above $4 \%$ as a thermal conductance-type hydrogen gas sensor based on the output voltage of thermocouple A when the hydrogen concentration is zero.

\section{Results}

\subsection{Output characteristics for injections of various hydrogen gas concentrations in air}

After we filled a metal container of about $30 \mathrm{~mL}$, in which the hydrogen sensor is placed, with pure air at a room temperature of $20^{\circ} \mathrm{C}$, hydrogen occluded in the $\mathrm{Pd}$ film was discharged by heating region A to about $150^{\circ} \mathrm{C}$ for $5 \mathrm{~s}$ with a power of $255 \mathrm{~mW}$ applied to the microheater formed on the cantilever of the sensor device shown in Fig. 1. Then, we filled the container with hydrogen gas of concentrations of $0,1,5,10,20,60$, and $100 \%$ relative to air by slowly injecting it over 2.5 s. In Fig. 4 , the output voltage 


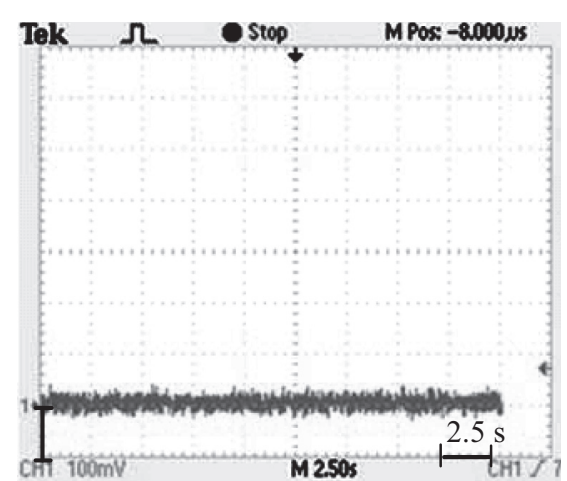

(a) $\mathrm{H}_{2}$ : 0 vol. $\%, 100 \mathrm{mV} / \mathrm{div}$.

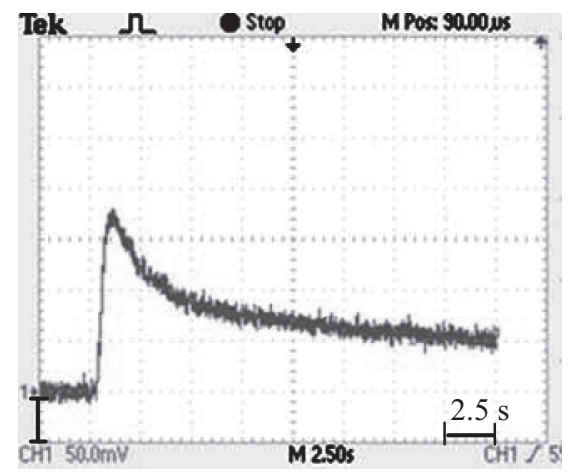

(c) $\mathrm{H}_{2}: 5$ vol. $\%, 50 \mathrm{mV} /$ div.

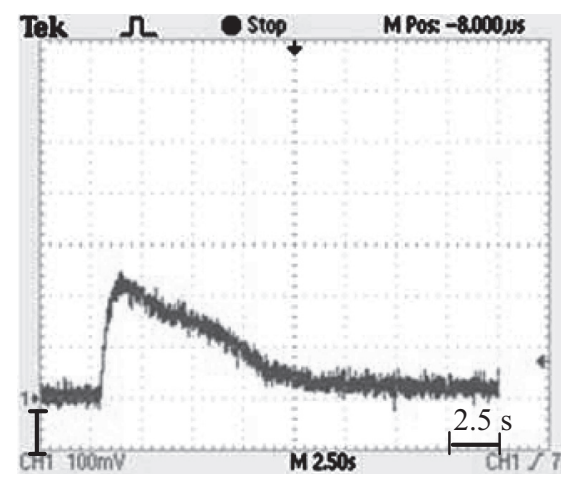

(e) $\mathrm{H}_{2}: 20$ vol. $\%, 100 \mathrm{mV} /$ div.

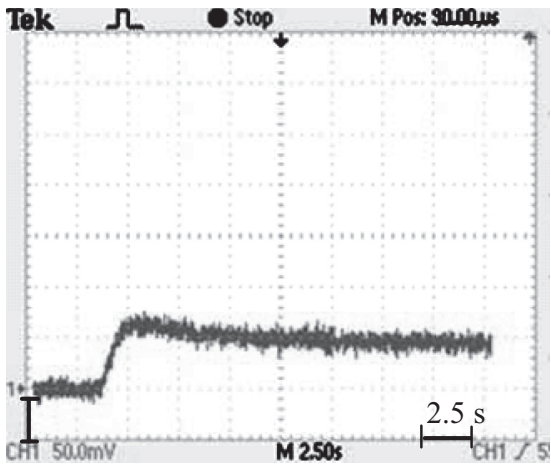

(b) $\mathrm{H}_{2}: 1$ vol. $\%, 50 \mathrm{mV} /$ div.

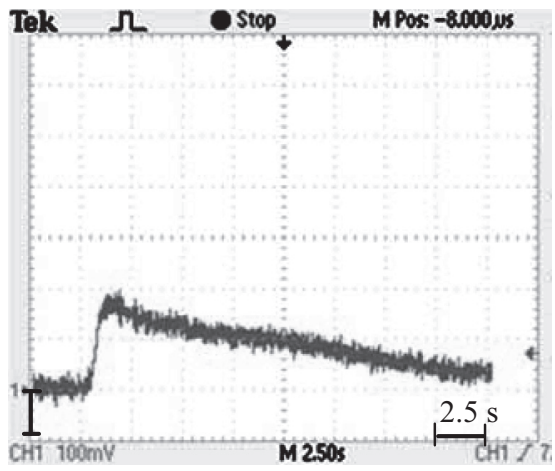

(d) $\mathrm{H}_{2}: 10$ vol. $\%, 100 \mathrm{mV} /$ div.

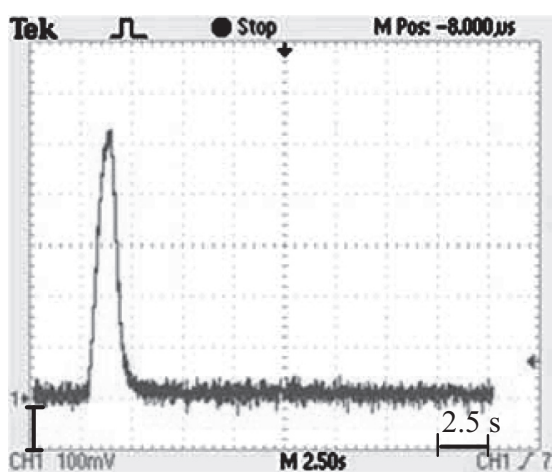

(f) $\mathrm{H}_{2}: 100$ vol. $\%, 100 \mathrm{mV} /$ div.

Fig. 4. Output voltage waveforms corresponding to the temperature difference $\Delta T b$ when various hydrogen gas concentrations diluted by pure air are injected. 
waveforms are shown when we have measured the temperature difference $\Delta T b$ under the above-mentioned conditions. The horizontal axis is all $2.5 \mathrm{~s} /$ div. As for the vertical axis, we have adopted a unit of $100 \mathrm{mV} /$ div. for 0 and $100 \%$ hydrogen concentrations to compare them at the initial setting. However, for the other hydrogen concentration, a unit of 50 or $100 \mathrm{mV} / \mathrm{div}$. is adopted depending on the output intensity.

We have ascertained that no influence of the flow during gas injection was observed at all because of the fact that no output change is observed during injection of $0 \%$ hydrogen concentration (pure air). It is understood that a wavy peak grows as the hydrogen concentration is increased; moreover, the wavy peak width broadens in a low hydrogen concentration.

In this paper, we will not describe the detailed characteristics of the hydrogen concentration in pure nitrogen gas; however, we want to add that a similar tendency in air is also observed in pure nitrogen gas. Therefore, we can measure the hydrogen concentration even in the state without oxygen.

\subsection{Output characteristics when hydrogen gas of $100 \%$ is injected at various ambient temperatures}

The above-mentioned container of about $30 \mathrm{~mL}$ is filled with $100 \%$ hydrogen gas by slow injection over $5 \mathrm{~s}$, after the container, which is put in a constant-temperature tank set at various ambient temperatures, is filled with pure air. In Fig. 5, output voltage waveforms depending on the temperature difference of $B_{S}$ and $B_{R}$ at various ambient temperatures are shown, where the unit of the horizontal axis is $5 \mathrm{~s} /$ div. It is understood that the shape of the waveforms sharpens and the peak increases as the ambient temperature increases.

\subsection{Output characteristics of low-concentration hydrogen gas}

After the injection of hydrogen gas of various concentrations has been completed, the microheater is heated in the square wave voltage for $5 \mathrm{~s}$ by an electric power of $255 \mathrm{~mW}$. Output voltage waveforms corresponding to the temperature difference of the $B_{S}$ and $B_{R}$ regions for various low hydrogen concentrations are shown in Fig. 6. The time when the heating of the microheater began is graphed as 0 in this figure. The temperature of region A during heating is estimated to be about $150^{\circ} \mathrm{C}$. The detection of an extremely small amount of hydrogen gas becomes important because hydrogen is an explosion hazard when its concentration in air becomes about $4 \%$.

In the measurement of a low hydrogen concentration of $4 \%$ or less and that of a high hydrogen concentration of $4 \%$ or more, different methods are used. In the heating period in this low hydrogen gas concentration, the temperature rise is obtained as a result of diverse effects existing together, such as exothermic effects based on the occlusion of hydrogen and the catalyst in the Pd film, and cooling effects based on heat conduction depending on the hydrogen concentration, etc. as the concentration of hydrogen gas increases. However, it is observed that the effect of heat radiation on the thermal conductivity of hydrogen gas increases when the hydrogen gas concentration exceeds $4 \%$, and the output voltage becomes small. That is, the output voltage of the hydrogen gas concentration of 5\% has become smaller than that of 4\%, as shown in Fig. 6. 


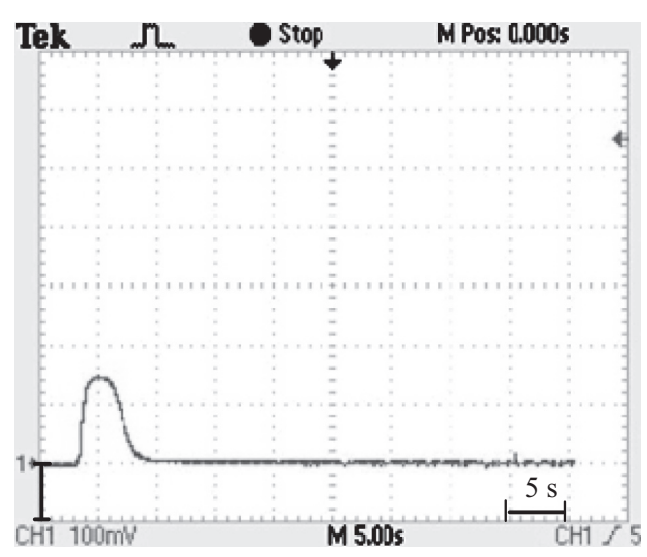

(a) $20^{\circ} \mathrm{C}, 100 \mathrm{mV} / \mathrm{div}$.

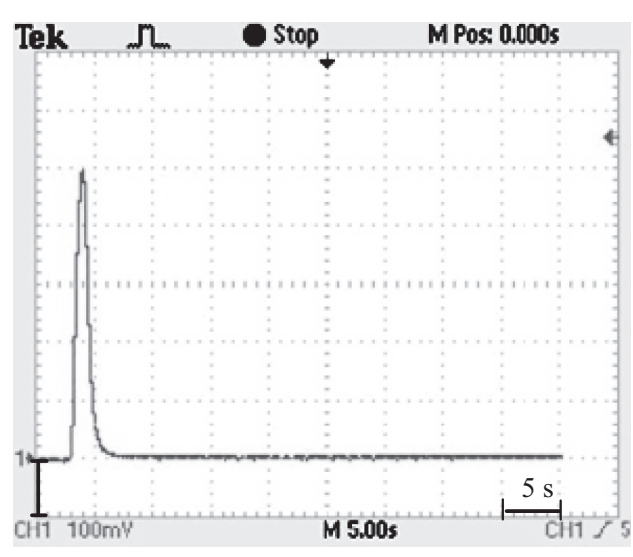

(b) $40^{\circ} \mathrm{C}, 100 \mathrm{mV} / \mathrm{div}$.

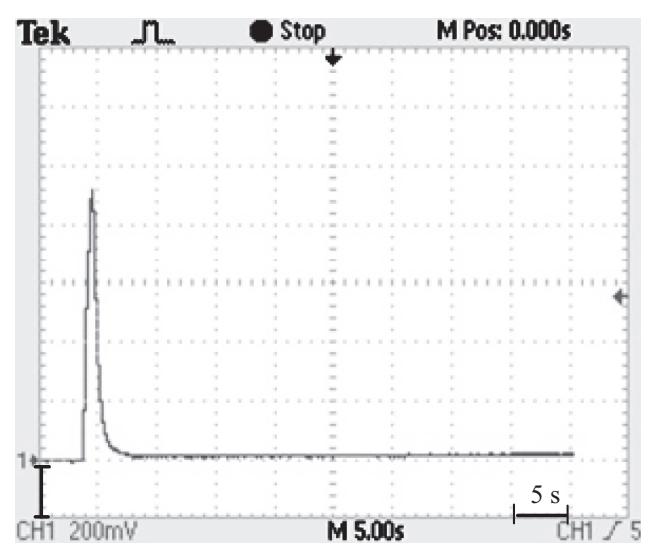

(c) $60^{\circ} \mathrm{C}, 200 \mathrm{mV} / \mathrm{div}$.

Fig. 5. Output voltage waveforms depending on temperature difference $\Delta T b$ in various ambient temperatures.

When the temperature difference $\Delta T b$ between regions $\mathrm{B}_{\mathrm{S}}$ and $\mathrm{B}_{\mathrm{R}}$, which has a slight difference in shape with region $\mathrm{B}_{\mathrm{S}}$, is measured during the heating-on cycle, there is a problem in that the output appears even if the hydrogen concentration is 0 . Therefore, we consider that the error margin may be large in the measurement of an extremely small concentration of hydrogen gas.

To solve the above-mentioned problem, the temperature difference $\Delta T b$ of the sensing area and reference area should be measured during the cooling cycle after the heating of the microheater is stopped. Figure 7 shows the magnified output voltage waveforms in an extremely low hydrogen gas concentration range $(0.5 \%$ or less $)$ among the 


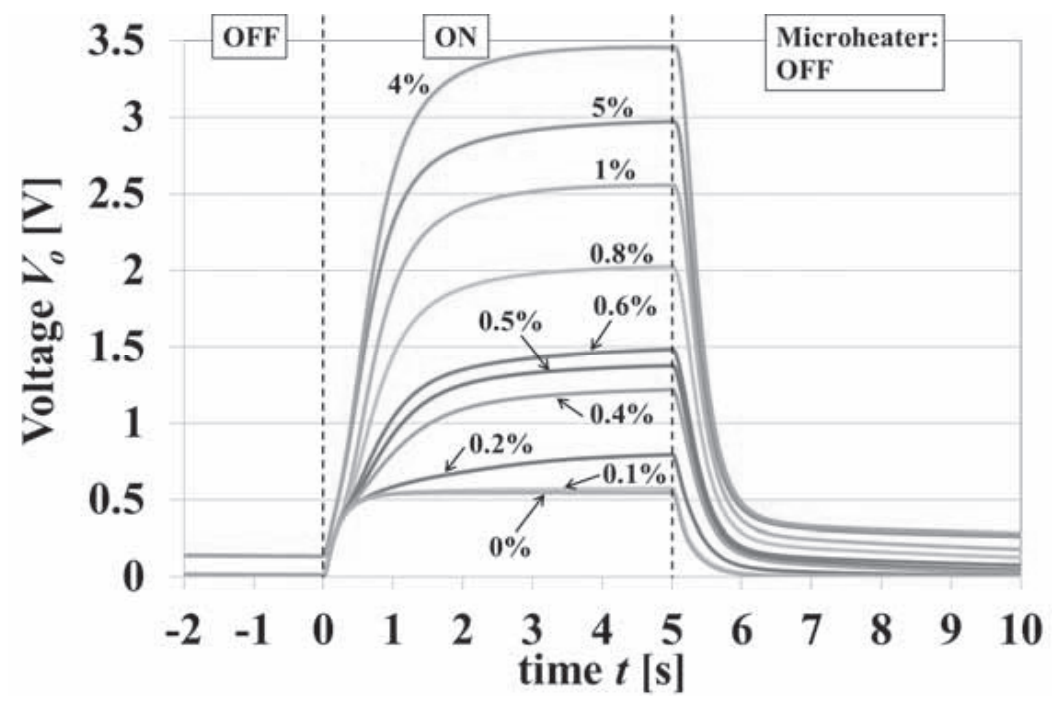

Fig. 6. Output voltage waveforms corresponding to the temperature difference $\Delta T b$ for various low hydrogen concentrations.

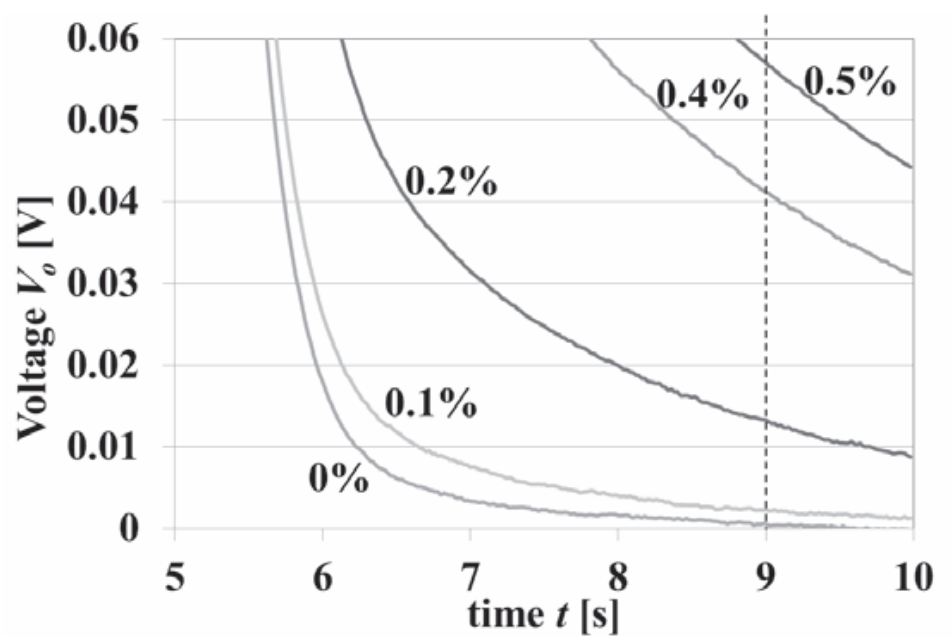

Fig. 7. Magnified output voltage waveforms in an extremely low hydrogen gas concentration range $(0.5 \%$ or less $)$ during the cooling process after the heating is stopped. 
characteristics shown in Fig. 6 during the cooling process after the heating has stopped. In a low hydrogen concentration, it is understood that the temperature of the sensing area is still above room temperature because of hydrogen occlusion, whereas the temperature of the reference area completely returns to room temperature at a time of about $8 \tau$, where $\tau$ is a thermal time constant (about $500 \mu$ s in this prototype sensor) of the cantilevertype microheater, measured after the heating is stopped (at about $4 \mathrm{~s}$ after the heating is stopped). This temperature difference $\Delta T b$ rises with an increase in the hydrogen concentration in this low-concentration area. Since the temperature of the sensing area should be room temperature when the hydrogen concentration is 0 , the null detection method with extremely high accuracy and high sensitivity can be used to measure the temperature difference $\Delta T b$ of the sensing area and reference area at room temperature. Therefore, an extremely highly accurate measurement of the hydrogen concentration becomes possible for this hydrogen gas sensor.

Figure 8 shows a graph of the output voltage in a hydrogen concentration of less than $1 \%$ at the time indicated by the dotted line shown in Fig. 7. The dotted line in Fig. 7, which is lined at $9 \mathrm{~s}$ in the horizontal axis, is at $4 \mathrm{~s}$ later $(0.5 \times 8 \mathrm{~s})$ since the heater was turned off.

In our experiments, the mixing ratio of air and hydrogen gas was adjusted by using the gas flow meter. Therefore, we could calibrate the hydrogen concentration only above $0.1 \%$.

\subsection{Output characteristics of high-concentration hydrogen gas}

In hydrogen gas concentration above $4 \%$, the temperature difference between the substrate and region $\mathrm{A}$, in which the microheater is formed, of the hydrogen gas sensor as shown in Fig. 1 is measured using thermocouple A as a thermal conductance-type hydrogen gas sensor, which is based on the fact that hydrogen gas has the highest thermal

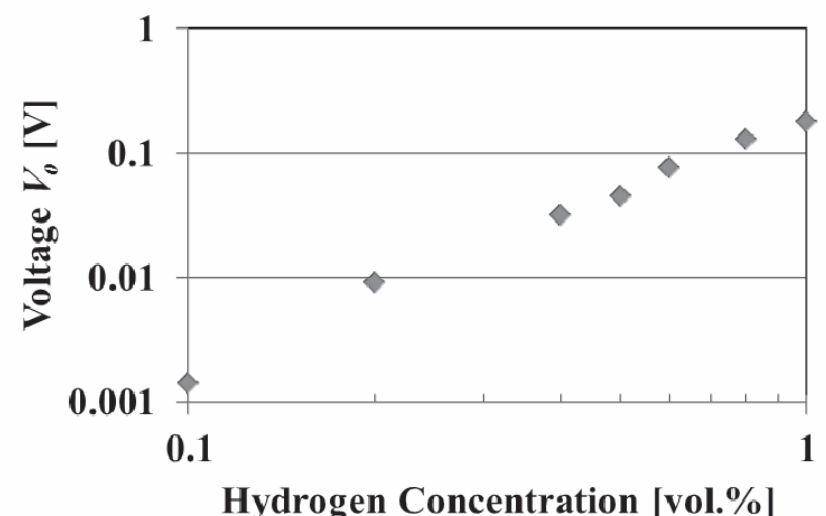

Fig. 8. Graph of the output voltage $V_{0}$ of the hydrogen concentration in less than 1 vol.\% at the time indicated by the dotted line shown in Fig. 7. 
conductivity of all gases. Figure 9 shows the output voltage waveform of the hydrogen gas sensor for each hydrogen gas concentration when working in the high-hydrogenconcentration mode, and Fig. 10 shows the output characteristics of the hydrogen gas sensor for each hydrogen gas concentration at that time, based on the output voltage of $0 \%$ hydrogen gas concentration.

\section{Discussion}

In Fig. 4, it is thought that a wavy peak will grow as the hydrogen concentration is raised due to an increase in the caloric value, because the hydrogen occlusion reaction per unit time increases as the hydrogen gas concentration increases. A peak in the output voltage will appear when the hydrogen occlusion in the Pd film reaches saturation and the exothermic reaction stops, and then the temperature begins to fall. When the hydrogen concentration is low, the quantity of hydrogen occlusion per unit time to the $\mathrm{Pd}$ film becomes small and the time until saturation will be extended. This fact may be the reason why the waveform becomes wide when the hydrogen concentration is low.

In contrast, when the hydrogen concentration is high, a sharp peak and large height can be expected because hydrogen occlusion is achieved in a short time and the exothermic reaction is also quickly finished.

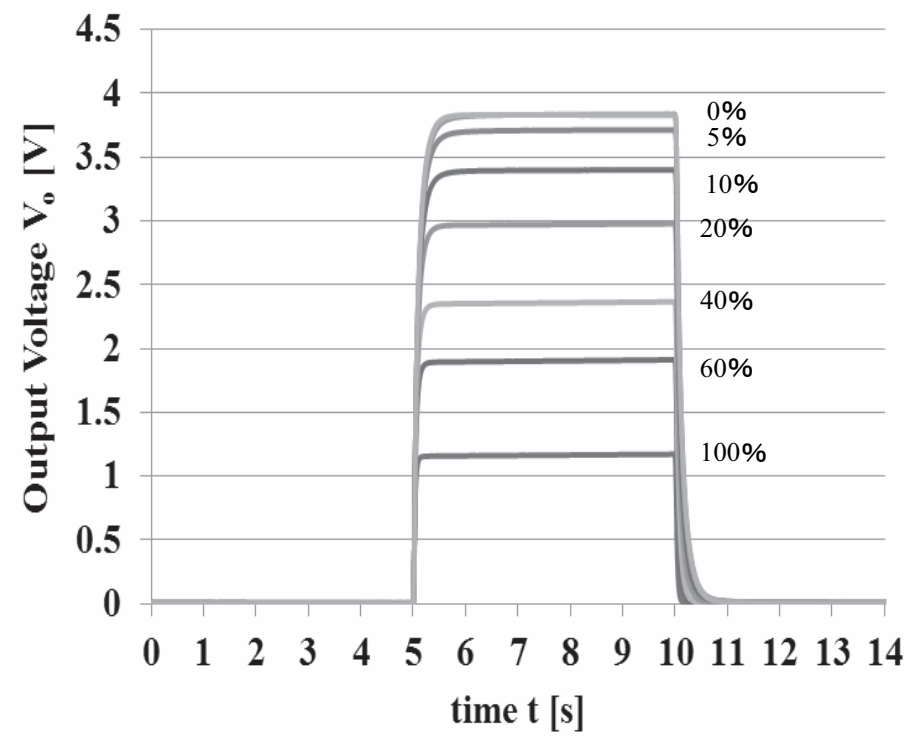

Fig. 9. Output voltage waveform of the hydrogen gas sensor for each hydrogen gas concentration when working in the high-hydrogen-concentration mode. 


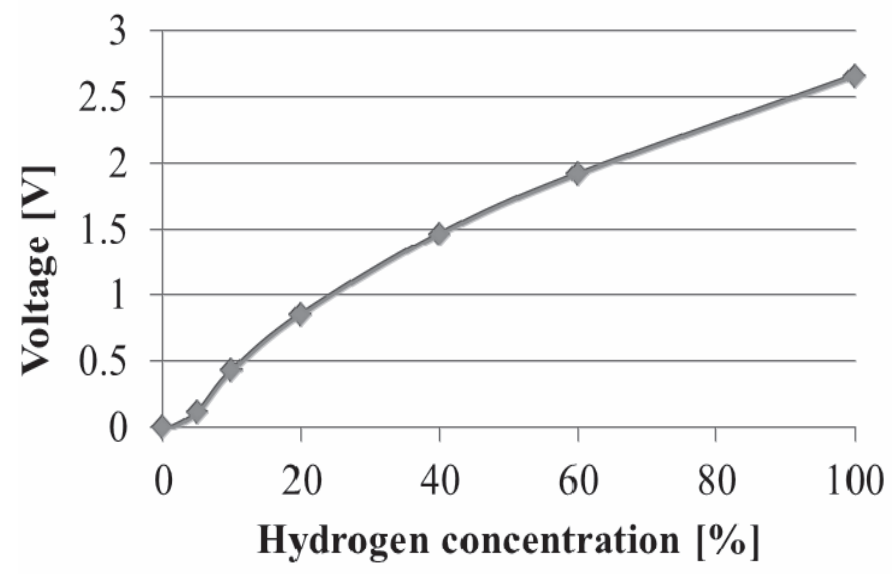

Fig. 10. Output characteristics of the hydrogen gas sensor to each hydrogen gas concentration based on the output voltage of 0 vol.\% hydrogen gas concentration.

In general, if the length of a cantilever becomes half, it is understood that the thermal time constant becomes $1 / 4$. Therefore, it is thought that the high-speed detection of less than $1 \mathrm{~s}$ is enabled by the miniaturization of the cantilever of our proposed hydrogen sensor.

In Fig. 5, it is thought that, in air, the reason why the shape of waves sharpens and the output peak grows as the ambient temperature rises is that the reaction of the injected hydrogen gas with oxygen adsorbed on the Pd surface becomes active as the temperature increases. The output peak is observed to grow as the ambient temperature decreases in pure nitrogen gas, although it is not shown here.(7) Because a temperature rise was observed in a pure nitrogen gas atmosphere by injection of hydrogen gas, it is understood that these phenomena must be based on an occlusion effect of hydrogen and that oxygen is not necessary for this reaction.

Figure 11 shows the output voltage of the hydrogen gas sensor when the container was filled with pure methane $\left(\mathrm{CH}_{4}\right)$ gas as a flammable gas by injection over $2.5 \mathrm{~s}$ after the container was substituted by pure nitrogen (a) or air (b). It is understood that the output voltage, when methane gas is injected, is not observed at all with the same hydrogen gas sensor (as shown in Figs. 4 and 5). We may conclude that the methane gas did not respond at all to our hydrogen gas sensor when working at the low-hydrogenconcentration mode. From these results, as for this hydrogen sensor, it is confirmed that there is a high selectivity for hydrogen but no response to methane gas, because of the method using the effect of the exothermic reaction based on hydrogen occlusion of $\mathrm{Pd}$ film.

From Fig. 7, it is understood that the output voltage does not vanish even if it passes by several times the thermal time constant after the heating of the heater stops in the 


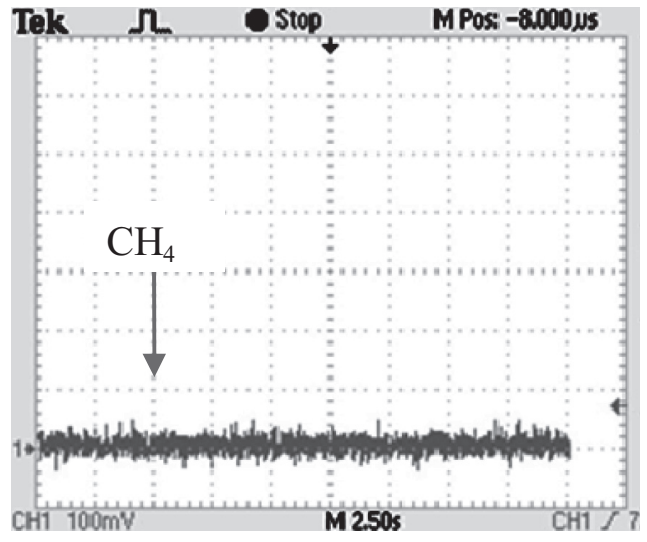

(a) in nitrogen gas

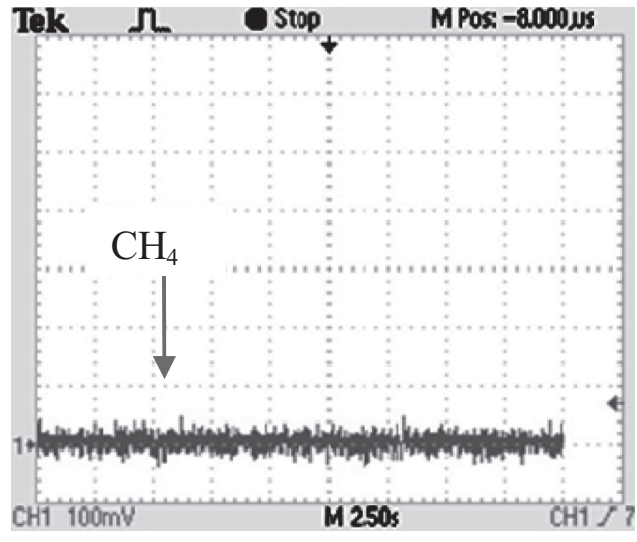

(b) in air

Fig. 11. Output voltage of the hydrogen gas sensor when the container was filled with pure methane $\left(\mathrm{CH}_{4}\right)$ gas (H: $2.5 \mathrm{~s} /$ div., $\mathrm{V}: 100 \mathrm{mV} /$ div. $)$ (a) in nitrogen gas and (b) in air.

atmosphere of various hydrogen gas concentrations. These facts will come from the exothermic reaction due to the discharged hydrogen gas during the heater-on state and the reocclusion during the cooling state after the heating stops. This may be clear from the fact that the time until hydrogen is saturated by hydrogen occlusion becomes longer as the hydrogen concentration decreases, as shown in Fig. 4.

In Fig. 7, the mixing ratio of hydrogen gas with pure air to measure the hydrogen concentration was determined by calibration using the gas flow meter; however, its accuracy of calibration was $0.1 \%$. Therefore, we could not measure the hydrogen concentration of less than $0.1 \%$. It is thought that the measurement of at least 100 ppm $(0.01 \%)$ is possible even using this present hydrogen gas sensor if we use a more accurate calibration, because we can use the null detection method for our hydrogen sensor.

In combustion-type hydrogen gas sensors using catalysis, it is necessary to heat the sensing area and to measure the hydrogen concentration during its heating. However, the temperature difference $\Delta T b$ between the reference area and sensing area will surely exist during heating owing to a minute difference in their thermal capacity and conductance, and it is hard to compensate for this temperature difference completely. Therefore, in these types of sensor, it is essentially hard to measure a hydrogen concentration of about $0 \%$.

On the other hand, a hydrogen concentration in the vicinity of $0 \%$ can be measured in detail using our proposed hydrogen gas sensor because the concentration measurement is carried out during the cooling process and by the null method, which is that the output voltage essentially becomes 0 when the hydrogen concentration is $0 \%$.

Sensing in the low-hydrogen-concentration mode that uses the exothermic reaction 
based on occlusion of the Pd film and sensing in the high-hydrogen-concentration mode using the thermal conductivity of hydrogen can be carried out at the same time using only one hydrogen gas sensor during a heating cycle.

In the low-concentration hydrogen gas, it takes a lot of time to return to the baseline at $0 \%$, as shown in Figs. 3 and 6 . However, we have experimentally confirmed that characteristic waveforms are almost stable and fixed when the heating cycle of heatingon for $5 \mathrm{~s}$ and heating-off for $5 \mathrm{~s}$ is repeated. The heating-on cycle up to about $150^{\circ} \mathrm{C}$ is used to refresh the sensor by discharging the occluded hydrogen from the Pd film.

\section{Conclusions}

We have proposed a new type of hydrogen gas sensor that is a combination of two types of hydrogen sensor, in which one is for a lower hydrogen gas concentration of $4 \%$ or less (the low-hydrogen-concentration mode), and the other is for a higher hydrogen gas concentration of more than $4 \%$ (the high-hydrogen-concentration mode). The hydrogen gas sensor for low concentration works basically at room temperature based on the exothermic reaction of the Pd film formed on the cantilever and that for highconcentration works during heating to about $150^{\circ} \mathrm{C}$ as a thermal conductance-type sensor by using the high thermal conductivity of hydrogen gas.

We have demonstrated that the proposed hydrogen gas sensor can be used to measure hydrogen concentration in the range from 0.1 to $100 \%$ within about $10 \mathrm{~s}$. If we use a more accurate gas flow meter for calibration, detection of the concentration of about $0.01 \%$ (100 ppm) of hydrogen gas will be expected to be carried within about $10 \mathrm{~s}$.

It is confirmed that our hydrogen gas sensor working in the low-hydrogenconcentration mode has a high selectivity for hydrogen but no response to methane gas, because of the method using the effect of the exothermic reaction based on hydrogen occlusion of the Pd film.

Since this hydrogen gas sensor has a very small thermal capacitance and thermal conductance because of the use of the cantilever formed by MEMS technology, a quicker response and lower power consumption will be achieved by its further miniaturization. Studies on long term stability and hysteresis for various hydrogen concentrations are necessary.

\section{Acknowledgment}

This research was supported in part by a Grant-in-Aid for Scientific Research of A-Step of the Japan Science and Technology Agency (JST).

\section{References}

1 W. Shin, K. Tajima, Y. Choi, N. Izu, I. Matsubara and N. Murayama: Sens. Actuators, B 108 (2005) 455.

2 L. F. Houlet, W. Shin, K. Tajima, M. Nishibori, N. Izu, T. Itoh and I. Matsubara: Sens. Actuators, B 130 (2008) 200. 
3 I. Eisele, T. Doll and M. Burgmair: Sens. Actuators, B 78 (2001) 19.

4 K. Yokosawa, S. Nakano, Y. Goto and K. Tsukada: Sensor Symposium 2005 (IEEJ, Tokyo, 2005) p. 435.

5 K. Yokosawa, K. Saitoh, S. Nakano, Y. Goto and K. Tsukada: Sens. Actuators, B 130 (2008) 94.

6 K. Tsukada, M. Kariya, T. Yamaguchi et al.: Jpn. J. Appl. Phys. 49 (2010) 024206.

7 N. Takashima and M. Kimura: Sensor Symposium 2010 (IEEJ, Tokyo, 2010) p. 573.

8 R. A. Varin, T. Czujko and Z. S. Wronski: Nanomaterials for Solid State Hydrogen Storage (Springer, New York, 2009) p. 8.

\section{About the Authors}
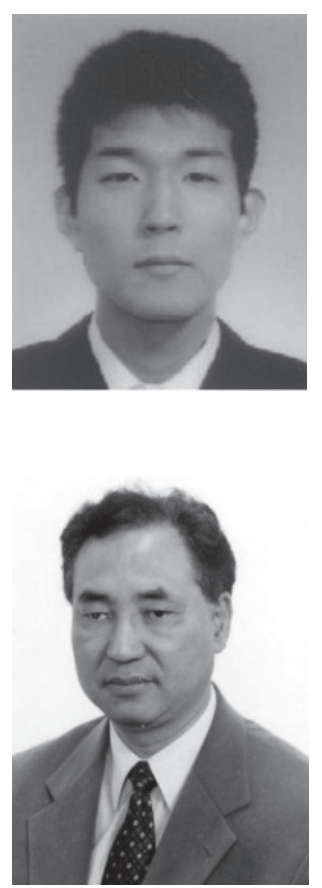

Noriaki Takashima was born in Yamagata, Japan in 1984. He received his B.S. and M.S. degrees in Electrical Engineering from Tohoku Gakuin University in 2006 and 2008, respectively, and currently is a graduate student of the same university. He is engaged in research on sensor devices, such as magnetic sensor, temperature sensor, pressure sensor, and hydrogen sensor. He is expected to be awarded a degree of Doctor of Engineering from Tohoku Gakuin University in March 2011. He is a member of the Institute of Electrical Engineers of Japan (IEEJ).

Mitsuteru Kimura was born in Akita, Japan in 1942. He received his B.S. degree in electronics from the University of Electro-Communications in 1967, and his M.S. and Ph.D. degrees also in electronics from Tohoku University in 1969 and 1974, respectively. He joined Tohoku Gakuin University in 1974, and is currently a Special Professor in the department of electronics. $\mathrm{He}$ is currently engaged in research on semiconductor devices, optical devices, and sensor devices. He is a member of IEEE, the Institute of Electrical Engineers of Japan (IEEJ), and the Japanese Society of Applied Physics (JSAP). 